\title{
Independence number of 2-factor-plus-triangles graphs
}

\author{
Jennifer Vandenbussche* and Douglas B. West ${ }^{\dagger}$ \\ Submitted: Jun 10, 2008; Accepted: Feb 18, 2009; Published: Feb 27, 2009 \\ Mathematics Subject Classification: 05C69
}

\begin{abstract}
A 2-factor-plus-triangles graph is the union of two 2-regular graphs $G_{1}$ and $G_{2}$ with the same vertices, such that $G_{2}$ consists of disjoint triangles. Let $\mathcal{G}$ be the family of such graphs. These include the famous "cycle-plus-triangles" graphs shown to be 3 -choosable by Fleischner and Stiebitz. The independence ratio of a graph in $\mathcal{G}$ may be less than $1 / 3$; but achieving the minimum value $1 / 4$ requires each component to be isomorphic to the 12-vertex "Du-Ngo" graph. Nevertheless, $\mathcal{G}$ contains infinitely many connected graphs with independence ratio less than $4 / 15$. For each odd $g$ there are infinitely many connected graphs in $\mathcal{G}$ such that $G_{1}$ has girth $g$ and the independence ratio of $G$ is less than $1 / 3$. Also, when 12 divides $n$ (and $n \neq 12$ ) there is an $n$-vertex graph in $\mathcal{G}$ such that $G_{1}$ has girth $n / 2$ and $G$ is not 3-colorable. Finally, unions of two graphs whose components have at most $s$ vertices are $s$-choosable.
\end{abstract}

\section{Introduction}

The Cycle-Plus-Triangles Theorem of Fleischner and Stiebitz [5] states that if a graph $G$ is the union of a spanning cycle and a 2-factor consisting of disjoint triangles, then $G$ is 3 -choosable, where a graph is $k$-choosable if for every assignment of lists of size $k$ to the vertices, there is a proper coloring giving each vertex a color from its list. Sachs [8] proved by elementary methods that all such graphs are 3-colorable. Both results imply an earlier conjecture by $\mathrm{Du}, \mathrm{Hsu}$, and Hwang [1], stating that a cycle-plus-triangles graph with $3 k$ vertices has independence number $k$. Erdös [3] strengthened the conjecture to the more well-known statement that these graphs are 3-colorable. We return to the original topic of independence number but study it on a more general family of graphs.

\footnotetext{
*Department of Mathematics, Southern Polytechnic State University, Marietta, GA 30060, jvandenb@spsu.edu

${ }^{\dagger}$ Department of Mathematics, University of Illinois, Urbana, IL 61801, west@math.uiuc.edu. Research partially supported by the National Security Agency under Award No. H98230-06-1-0065.
} 
A 2-factor-plus-triangles graph is a union of two 2-regular graphs $G_{1}$ and $G_{2}$ on the same vertex set, where the components of $G_{2}$ are triangles. Note that $G_{1}$ and $G_{2}$ may share edges. For such a graph $G$, we denote the vertex sets of the components of $G_{2}$ as $T_{1}, \ldots, T_{k}$, with $T_{x}=\left\{x_{1}, x_{2}, x_{3}\right\}$, and we refer to $T_{x}$ as a "triple" to distinguish it from a 3-cycle in $G_{1}$. When $G_{1}$ is a single cycle, $G$ is a cycle-plus-triangles graph.

Let $\mathcal{G}$ denote the family of 2 -factor-plus-triangles graphs. It is easy to construct graphs in $\mathcal{G}$ that contain $K_{4}$ (see Figure 1, for example), so graphs in $\mathcal{G}$ need not be 3 -colorable. Erdos [3] asked if a graph in $\mathcal{G}$ is 3 -colorable whenever its factor $G_{1}$ is $C_{4}$-free. Fleischner and Stiebitz [6] answered this negatively, citing an infinite family of such graphs in $\mathcal{G}$ that are 4-critical, due to Gallai. In fact, graphs in $\mathcal{G}$ with $3 k$ vertices may fail to have an independent set of size $k$, such as the graph in Figure 1 due to Du and Ngo [2]. Here we draw only $G_{1}$ and indicate the triples $T_{a}, T_{b}, T_{c}, T_{d}$ using subscripted indices.
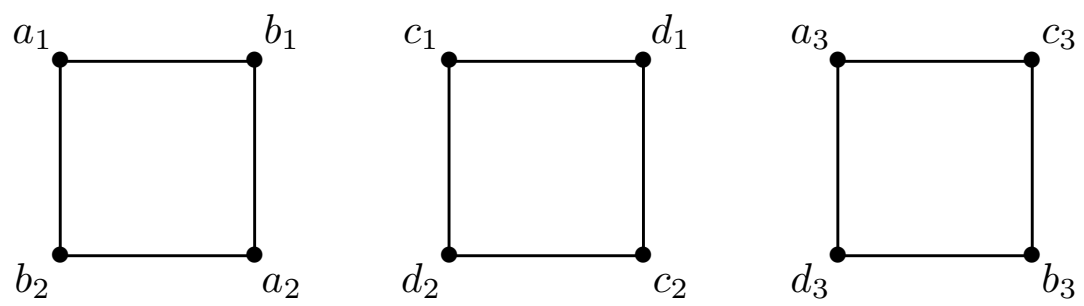

Figure 1: The Du-Ngo graph $G_{D N}$, omitting triangles on sets of the form $\left\{x_{1}, x_{2}, x_{3}\right\}$.

An independent set is a set of pairwise nonadjacent vertices. The independence number $\alpha(G)$ of a graph $G$ is the maximum size of such a set in $G$.

Proposition 1.1. The independence number of the Du-Ngo graph $G_{D N}$ is 3 .

Proof. An independent set $S$ in $G_{D N}$ contains at most one vertex from each of the 4-cliques $\left\{a_{1}, b_{1}, a_{2}, b_{2}\right\}$ and $\left\{c_{1}, d_{1}, c_{2}, d_{2}\right\}$. Further, $S$ contains two vertices of $\left\{a_{3}, b_{3}, c_{3}, d_{3}\right\}$ only if it avoids one of the 4 -cliques. Thus $|S| \leq 3$, and $\left\{a_{1}, c_{1}, d_{3}\right\}$ achieves the bound.

The independence ratio of an $n$-vertex graph $G$ is $\alpha(G) / n$. Proposition 1.1 states that the independence ratio of $G_{D N}$ is $1 / 4$. Because graphs in $\mathcal{G}$ have maximum degree at most 4 and do not contain $K_{5}$, Brooks' Theorem implies that every graph in $\mathcal{G}$ has independence ratio at least $1 / 4$. We characterize the graphs achieving equality in this easy bound; they are those in which every component is $G_{D N}$. We produce larger independent sets for all other graphs in $\mathcal{G}$. We also construct infinitely many connected graphs in $\mathcal{G}$ with independence ratio less than $4 / 15$. However, we conjecture that for any $t$ less than $4 / 15$, only finitely many connected graphs in $\mathcal{G}$ have independence ratio at most $t$.

In light of Erdős' question about 3-colorability of graphs in $\mathcal{G}$ when $G_{1}$ has no 4 cycle, we study the independence ratio under girth restrictions for $G_{1}$. For any odd $g$, we construct infinitely many connected examples in which the girth of $G_{1}$ is $g$ and yet 
the independence ratio is less than $1 / 3$; it can be as small as $\frac{1}{3}-\frac{1}{g^{2}+2 g}$ when $g \equiv 1$ mod 6 . The number of vertices in each example is more than $g^{2}$, and we conjecture that the independence ratio of $G$ is $1 / 3$ when $G_{1}$ has girth at least $\sqrt{|V(G)|}$. On the other hand, no girth threshold less than $|V(G)|$ can guarantee 3-colorability; when the number of vertices is a nontrivial multiple of 12 , we construct examples where $G_{1}$ consists of just two cycles of equal length but $G$ is not 3-colorable.

Finally, we show that if $G$ is a union of two graphs whose components have at most $s$ vertices, then $G$ is $s$-choosable; this yields 3 -choosability for graphs in $\mathcal{G}$ where the components of $G_{1}$ are all 3 -cycles. This last result is an easy consequence of the $s$ choosability of the line graphs of bipartite graphs.

Our graphs have no multiple edges; when $G_{1}$ and $G_{2}$ share an edge, its vertices have degree less than 4 in the union. For a graph $G$ and a vertex $x \in V(G)$, the neighborhood $N_{G}(x)$ is the set of vertices adjacent to $x$ in $G$, and a $G$-neighbor of $x$ is an element of $N_{G}(x)$. For $S \subseteq V(G)$, we let $N_{G}(S)=\bigcup_{x \in S} N_{G}(x)$. If $A$ and $B$ are sets, then $A-B=\{a \in A: a \notin B\}$.

\section{Independence ratio at least $1 / 4$}

The independence number of a graph is the sum of the independence numbers of its components. Therefore, to characterize the graphs in $\mathcal{G}$ with independence ratio $1 / 4$, it suffices prove that every connected graph in $\mathcal{G}$ other than $G_{D N}$ has independence ratio larger than $1 / 4$. Let $\mathcal{G}^{\prime}=\left\{G \in\left(\mathcal{G}-\left\{G_{D N}\right\}\right): G\right.$ is connected $\}$.

Proving this is surprisingly difficult. We present an algorithm to produce a sufficiently large independent set for any $G \in \mathcal{G}^{\prime}$. A simple greedy algorithm finds an independent set with almost $1 / 4$ of the vertices; it will be applied to prove the full result. This simple algorithm maintains an independent set $I$ and the set $S$ of neighbors of $I$.

Algorithm 2.1. Given an independent set $I$ in $G$, let $S=N_{G}(I)$. While $I \cup S \neq V(G)$, choose $v \in V(G)-(I \cup S)$ to minimize $|N(v)-S|$, and add $v$ to $I$ and $N_{G}(v)$ to $S$.

Lemma 2.2. If $G$ is an n-vertex graph in $\mathcal{G}^{\prime}$, then $\alpha(G) \geq(n-1) / 4$. If $G$ has an independent set $I_{0}$ with $3\left|I_{0}\right|>\left|N_{G}\left(I_{0}\right)\right|$, then $\alpha(G)>n / 4$.

Proof. Initialize Algorithm 2.1 with $I$ as any single vertex in $G$; this puts at most 4 vertices in $S$. At each subsequent step, some vertex $v$ outside $I \cup S$ has a neighbor in $S$, since $G$ is connected and $N_{G}(I)=S$. Hence each step adds at most 3 vertices to $S$ and 1 vertex to $I$. Therefore, $|S| \leq 3|I|+1$ when the algorithm ends. Since $n=|I|+|S|$ at that point, we conclude that $|I| \geq(n-1) / 4$.

If $3\left|I_{0}\right|>\left|N_{G}\left(I_{0}\right)\right|$, then initializing Algorithm 2.1 with $I=I_{0}$ (and $S=N_{G}\left(I_{0}\right)$ ) yields $|S| \leq 3|I|-1$ at the end by the same computation, and hence $|I| \geq(n+1) / 4$. 
In order to push the independence ratio above 1/4, we will preface Algorithm 2.1 with another algorithm that will choose the initial independent set more carefully, seeking an independent set $I_{0}$ as in Lemma 2.2 or one that will lead to a gain later under Algorithm 2.1.

First we characterize how 4-cliques can arise in graphs in $\mathcal{G}$ (a $k$-clique is a set of $k$ pairwise adjacent vertices).

Lemma 2.3. A 4-clique in a graph $G$ in $\mathcal{G}$ arises only as the union of a 4 -cycle in $G_{1}$ and disjoint edges from two triples in $G_{2}$ (Figure 2 below shows such a 4-clique).

Proof. Let $X$ be a 4-clique in $G$. Since $G_{1}$ contributes at most two edges to each vertex, each vertex in $X$ has a $G_{2}$-neighbor in $X$. In particular, no triple in $G_{2}$ is contained in $X$, and $X$ must have the form $\left\{a_{1}, a_{2}, b_{1}, b_{2}\right\}$ for some $T_{a}$ and $T_{b}$. To make $X$ pairwise adjacent, $a_{1}, b_{1}, a_{2}, b_{2}$ in order must form a 4-cycle in $G_{1}$.

We define a substructure that yields a good independent set for the initialization of Algorithm 2.1. A bonus 4-clique in a graph in $\mathcal{G}$ is a 4-clique $Q$ such that for some triple $T_{a}$ contributing two vertices to $Q$, the vertices of $N_{G_{1}}\left(a_{3}\right)$ lie in the same triple. Figure 2 illustrates the definition.
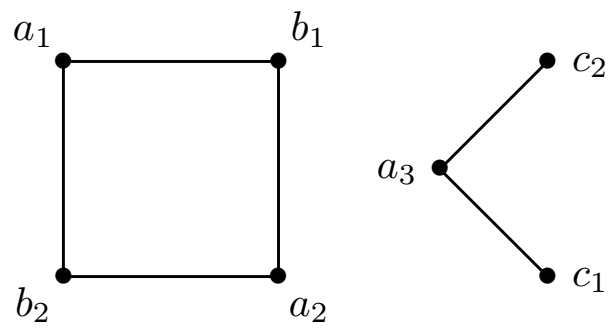

Figure 2: A bonus 4-clique

Lemma 2.4. If an $n$-vertex graph $G$ in $\mathcal{G}^{\prime}$ has a bonus 4 -clique, then $\alpha(G)>n / 4$.

Proof. Consider a bonus 4-clique, labeled as in Figure 2 without loss of generality. The set $\left\{b_{1}, a_{3}, c_{3}\right\}$ is independent, and its neighborhood is $\left\{a_{1}, a_{2}, b_{2}, b_{3}, c_{1}, c_{2}\right\} \cup N_{G_{1}}\left(c_{3}\right)$. Thus setting $I_{0}=\left\{b_{1}, a_{3}, c_{3}\right\}$ in Lemma 2.2 yields the conclusion.

A block of a graph is a maximal subgraph that contains no cut-vertex. Two blocks in a graph share at most one vertex, and a vertex in more than one block is a cut-vertex. A leaf block of a graph $G$ is a block that has at most one vertex shared with other blocks of $G$. We need a structural result to extract large independent sets from leaf blocks.

Lemma 2.5. Let $G$ be an n-vertex 4-regular graph in $\mathcal{G}^{\prime}$. If $G$ has no 4 -clique, then $G$ has an independent set $I$ such that $3|I|>\left|N_{G}(I)\right|$ or such that $3|I|=\left|N_{G}(I)\right|$ and $|I|<n / 4$. 
Proof. Every vertex of $G$ lies in a triple, and every triple lies in a block of $G$. Since $G$ is 4-regular, a leaf block contains a triple and at least one more vertex. A shortest path joining two vertices of the triple that uses a vertex outside the triple yields an even cycle with at most one chord. (Note: Erdős, Rubin, and Taylor [4] showed by a harder proof that all 2-connected graphs other than complete graphs and odd cycles have such a cycle.)

An independent set $I$ with $|I|>n / 4$ vertices satisfies $3|I|>\left|N_{G}(I)\right|$ and hence suffices.

We may assume that $G$ has no 4-cycle, since $G$ has no 4-clique and a 4-cycle in $G$ with at most one chord has an independent set $I$ with $3|I|=\left|N_{G}(I)\right|$ and $|I|=2 \neq n / 4$ (note that $3 \mid n$ ). If $C$ is an even cycle in $G$ having at most one chord, then at least one of the two maximum independent sets in $C$ contains at most one vertex of such a chord and is independent in $G$. Let $I$ be such an independent set.

Since each vertex of $I$ has at least two neighbors on $C$ and at most two outside it, $3|I| \geq\left|N_{G}(I)\right|$. We have found the desired set $I$ unless $|I|=n / 4$. In this case, let $T=V(G)-V(C)$. If $I$ is not a maximal independent set, then $\alpha(G)>n / 4$, so we may assume that every vertex of $T$ has a neighbor in $I$. Since $I \subseteq V(C)$, each vertex in $I$ has at most two neighbors in $T$. Hence each vertex of $T$ has exactly one neighbor in $I$, and each vertex of $I$ has two neighbors in $T$ (and $C$ has no chord).

Let $u, v, w$ be three consecutive vertices on $C$, with $u, w \in I$. Let $\left\{x, x^{\prime}\right\}=N_{G}(u) \cap T$ and $\left\{y, y^{\prime}\right\}=N_{G}(w) \cap T$. If $x x^{\prime} \notin E(G)$, then replacing $u$ with $\left\{x, x^{\prime}\right\}$ in $I$ yields $\alpha(G)>n / 4$. Hence we may assume that $x x^{\prime} \in E(G)$, and similarly $y y^{\prime} \in E(G)$. If $v$ has a neighbor in $\left\{x, x^{\prime}, y, y^{\prime}\right\}$, then $G$ has a 4-cycle, which we excluded. Since $G$ has no 4-clique, some vertex in $\left\{x, x^{\prime}\right\}$ has a nonneighbor in $\left\{y, y^{\prime}\right\}$, say $x y \notin E(G)$. Now replacing $\{u, w\}$ with $\{v, x, y\}$ in $I$ yields $\alpha(G)>n / 4$.

We now present an algorithm to apply before Algorithm 2.1, as "preprocessing". The proof of Lemma 2.5 can be implemented as an algorithm used by Algorithm 2.6 when $G$ has no 4-clique. Like Algorithm 2.1, Algorithm 2.6 maintains an independent set $I \subseteq V(G)$ and the set $S$ of its neighbors. It produces a nonempty independent set $I$ such that $3|I|>|S|$ or such that $3|I|=|S|<3 n / 4$ and all vertices of 4-cliques lie in $I \cup S$.

After Algorithm 2.6, we apply Algorithm 2.1 starting with this set as $I$. Lemma 2.2 implies that if $3|I|>|S|$, then $\alpha(G)>n / 4$. We will show in Theorem 2.8 that if $3|I|=|S|$, then the exhaustion of the 4-cliques during Algorithm 2.6 will guarantee the existence of a step in Algorithm 2.1 in which $S$ gains at most two vertices. Thus again we will have $3|I|>|S|$ and $|I|>n / 4$ at the end.

To facilitate the description of Algorithm 2.6, we introduce several definitions. A triple having two vertices in a 4-clique is a clique-triple. Two clique-triples that contribute two vertices each to the same 4-clique (see Lemma 2.3) are mates. If $T_{a}$ intersects a 4-clique $Q$, but $I \cup S$ does not intersect $T_{a} \cup Q$, then $T_{a}$ is a free clique-triple.

Algorithm 2.6. Given an $n$-vertex graph $G$ in $\mathcal{G}^{\prime}$, initialize $I=S=\varnothing$. Maintain $S=N_{G}(I)$. When we "stop", the current set $I$ is the output. 
Suppose first that $G$ has no 4-clique. If $E\left(G_{1}\right) \cap E\left(G_{2}\right) \neq \varnothing$, then let $I$ consist of one endpoint of such an edge and stop. Otherwise, $G$ is 4-regular; let $I$ be an independent set produced by the algorithmic implementation of Lemma 2.5, and stop.

If $G$ has a bonus 4-clique, then define $I$ as in Lemma 2.4 and stop.

If $G$ has a 4-clique but no bonus 4-clique, then repeat the steps below until either $3|I|>|S|$ or $I \cup S$ contains all vertices of 4-cliques; then stop.

1. If a vertex outside $I \cup S$ has at most two neighbors outside $S$, add it to $I$ and stop.

2. If there is a free clique-triple $T_{a}$ with mate $T_{b}$ such that $S$ contains $b_{3}$ or some $G_{1}$-neighbor of $a_{3}$, then add $\left\{a_{3}, b_{1}\right\}$ to $I$ and stop.

3. Otherwise, let $T_{a}$ be a free clique-triple with mate $T_{b}$, and let $N_{G_{1}}\left(a_{3}\right)=\left\{c_{3}, d_{3}\right\}$. Since $G$ has no bonus 4-clique, $c \neq d$. If $\left\{c_{1}, d_{1}, c_{2}, d_{2}\right\}$ is not a 4 -clique in $G$, then add $\left\{a_{3}, b_{1}\right\}$ to $I$. If $\left\{c_{1}, d_{1}, c_{2}, d_{2}\right\}$ is a 4-clique in $G$, then add $\left\{a_{3}, b_{1}, c_{3}, d_{1}\right\}$ to $I$.

Lemma 2.7. For $G \in \mathcal{G}^{\prime}$, Algorithm 2.6 produces an independent set I with neighborhood $S$ such that $3|I|>|S|$ or such that $3|I|=|S|$ and $I \cup S$ contains all 4 -cliques in $G$.

Proof. First suppose $G$ has no 4-clique. If $G$ is 4-regular, then Algorithm 2.6 uses the construction of Lemma 2.5 to produce $I$ such that $3|I|>|S|$ or such that $3|I|=|S|$ and $|I|<n / 4$ (and hence $I \cup S \neq V(G)$ ). If $G$ is not 4-regular, then it finds such a set of size 1 .

If $G$ has a bonus 4-clique, then the independent set $I$ is as in the proof of Lemma 2.4, with $3|I|>|S|$.

Therefore, we may assume that $G$ has a 4-clique but no bonus 4-clique. In this case, the algorithm iterates Step 3 until it reaches a state where Step 1 or 2 applies or it runs out of free clique-triples.

To show that ending in Step 1 or 2 yields the desired conclusion, suppose that each instance of Step 3 maintains $3|I| \geq|S|$. In Step 1, we then add one vertex to $I$ and at most two to $S$. In Step 2, we add $\left\{a_{3}, b_{1}\right\}$ to $I$ and $\left\{a_{1}, a_{2}, b_{2}, b_{3}\right\} \cup N_{G_{1}}\left(a_{3}\right)$ to $S$, but $S$ already contains at least one of these six vertices.

Hence we must show that Step 3 maintains $3|I| \geq|S|$. To avoid getting stuck by running out of free clique-triples before absorbing all 4-cliques into $I \cup S$, also we must maintain that every 4-clique not contained in $I \cup S$ intersects a free clique-triple.

These two properties hold initially. Suppose that they hold when we enter an instance of Step 3. We have mates $T_{a}$ and $T_{b}$, with $T_{a}$ being free. Since Step 2 does not apply, $b_{3} \notin S$, so $T_{b}$ also is free. Since $G$ has no bonus 4-clique, $c \neq d$.

In the first case, $\left\{c_{1}, d_{1}, c_{2}, d_{2}\right\}$ is not a 4 -clique, and we add $\left\{a_{3}, b_{1}\right\}$ to $I$. This adds $\left\{a_{1}, a_{2}, b_{2}, b_{3}\right\} \cup N_{G_{1}}\left(a_{3}\right)$ to $S$, gaining six vertices. The 4 -clique $\left\{a_{1}, a_{2}, b_{1}, b_{2}\right\}$ has been absorbed. The vertices of other 4-cliques that might enter $I \cup S$ are those in $T_{c} \cup T_{d}$. Suppose that $\left\{c_{1}, c_{2}, x_{1}, x_{2}\right\}$ is a 4-clique, with $T_{x}$ the mate of $T_{c}$. If $T_{x}$ is not free before this instance of Step 3, then $x_{3} \in S$, but now Step 2 would have applied instead of Step 3, with $T_{c}$ as $T_{a}$ and $T_{x}$ as $T_{b}$. Since the addition to $I$ does not affect $x_{3}$, afterwards $T_{x}$ remains free. Similarly, the mate of $T_{d}$ remains free if $T_{d}$ is a clique-triple. 
In the second case, $\left\{c_{1}, d_{1}, c_{2}, d_{2}\right\}$ is a 4 -clique, and we add $\left\{c_{3}, d_{1}\right\}$ to $I$. This is an instance of the first case for the mates $T_{c}$ and $T_{d}$ unless $N_{G_{1}}\left(c_{3}\right)=\left\{a_{3}, b_{3}\right\}$. However, that requires $G=G_{D N}$, labeled as in Figure 1. Since $G \in \mathcal{G}^{\prime}$, we find a 4 -clique where the first case of Step 3 applies.

Theorem 2.8. For $G \in \mathcal{G}^{\prime}$, using the output of Algorithm 2.6 as initialization to Algorithm 2.1 produces an independent set having more than $1 / 4$ of the vertices of $G$.

Proof. By Lemma 2.2, we may assume that the output of Algorithm 2.6 is an independent set $I$ with neighborhood $S$ such that $3|I|=|S|$ and every 4-clique is contained in $I \cup S$. Furthermore, if $G$ has no 4-clique, then $I \cup S \neq V(G)$. To complete the proof, we show that with such an initialization, the final step of Algorithm 2.1 adds at most two vertices to $S$ (hence strict inequality holds at the end).

We claim that also $I \cup S \neq V(G)$ when $G$ has a 4-clique and Algorithm 2.6 ends with $3|I|=|S|$. We noted in the proof of Lemma 2.7 that ending in Step 1 or 2 yields $3|I|>|S|$, so ending with $3|I|=|S|$ requires ending in Step 3. On the last step, we have free mates $T_{a}$ and $T_{b}$, and we add $\left\{a_{3}, b_{1}\right\}$ to $I$ and $\left\{a_{1}, a_{2}, b_{2}, b_{3}\right\} \cup N_{G_{1}}\left(a_{3}\right)$ to $S$. If this exhausts $V(G)$, then $N_{G_{1}}\left(a_{3}\right)=V(G)-(I \cup S)-\left(T_{a} \cup T_{b}\right)$ before the final step. The other vertices of the triples containing the vertices of $N_{G_{1}}\left(a_{3}\right)$ are already in $S$. These two vertices lie in the same triple; otherwise, each has at most two neighbors outside $S$ before the last step, and Step 1 would apply. On the other hand, if they belong to the same clique, then $\left\{a_{1}, a_{2}, b_{1}, b_{2}\right\}$ is a bonus 4-clique, which would have been used at the start.

Hence we may assume that at least one vertex remains outside $I \cup S$ when we move to Algorithm 2.1. We claim that at most two vertices are added to $S$ in the final step of Algorithm 2.1. If three vertices are added to $S$, then let $x$ be the vertex added to $I$, with neighbors $u, v, w$ added to $S$. Choosing one of $\{u, v, w\}$ instead of $x$ would also add at least three vertices to $S$, since we chose $v$ to minimize $|N(v)-S|$. This implies that $\{u, v, w, x\}$ is a 4 -clique in $G$. This possibility is forbidden, since all vertices contained in 4-cliques are added to $I \cup S$ during Algorithm 2.2.

Corollary 2.9. Every 2-factor-plus-triangles graph has independence ratio at least 1/4, with equality only for graphs whose components are all isomorphic to $G_{D N}$.

\section{Constructions}

The Du-Ngo graph $G_{D N}$ is the only graph in $\mathcal{G}^{\prime}$ with independence ratio $1 / 4$. In this section, we construct a sequence of graphs with independence ratio less than 4/15.

Figure 3 shows a 27-vertex graph $G$ in $\mathcal{G}^{\prime}$ with $\alpha(G)=\frac{1}{4}(27+1)$. Note that $G$ is connected. An independent set $I$ has at most six vertices in the subgraph inside the dashed box (at most two from each "column" of 4-cycles). Also, $I$ has at most one vertex in the remaining 3-cycle $\left[x_{3}, y_{3}, z_{3}\right]$ in $G_{1}$. Hence $\alpha(G) \leq 7=(27+1) / 4$, and $\left\{a_{1}, b_{3}, c_{1}, d_{3}, e_{1}, f_{3}, x_{3}\right\}$ achieves the upper bound. 


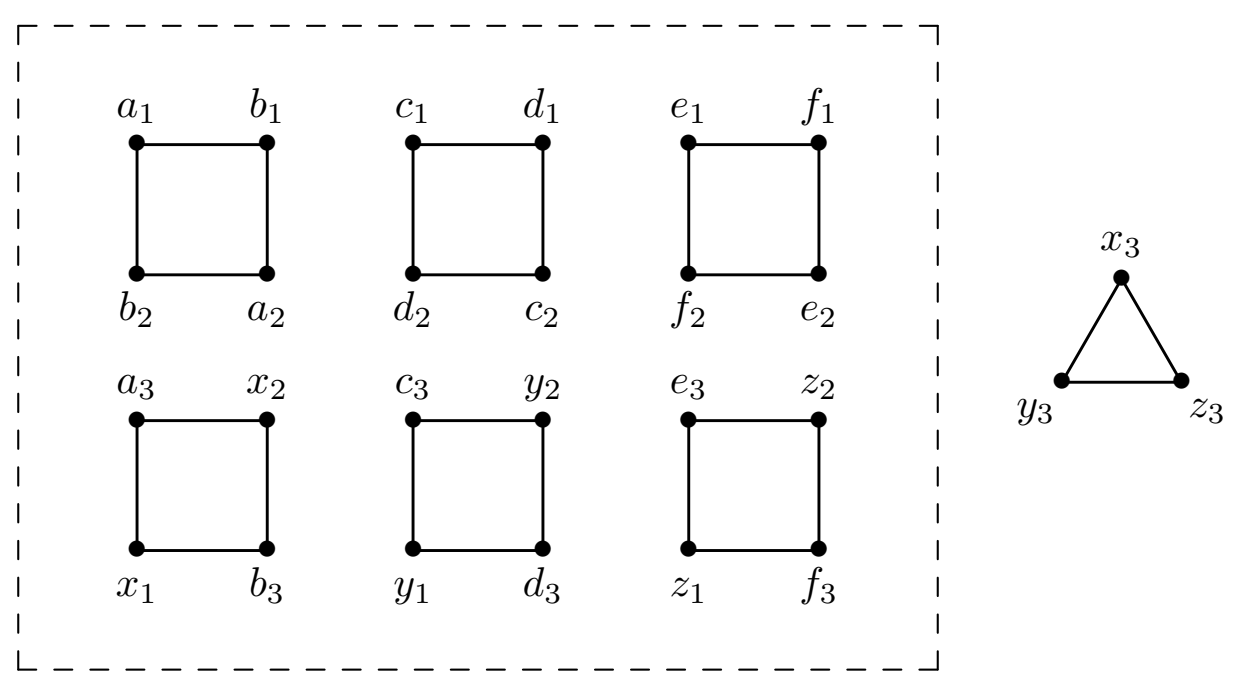

Figure 3: A graph in $\mathcal{G}^{\prime}$ with independence number $(n+1) / 4$

One may ask whether infinitely many graphs $G$ in $\mathcal{G}^{\prime}$ satisfy $\alpha(G)=(|V(G)|+1) / 4$, or at least with $\alpha(G) \leq(|V(G)|+c) / 4$ for some constant $c$. We conjecture that no such constant exists; in fact, we conjecture the following stronger statement.

Conjecture 3.1. For every $t<4 / 15$, only finitely many graphs in $\mathcal{G}^{\prime}$ have independence ratio at most $t$.

This conjecture is motivated by the following theorem, which shows that the conclusion is false when $t \geq 4 / 15$. To avoid confusion with our earlier use of $G_{1}$ and $G_{2}$, we use $Q_{i}$ and $R_{i}$ to index sequences of special graphs in this construction.

Theorem 3.2. For $i \geq 0$, there is a graph $Q_{i} \in \mathcal{G}$ with independence ratio $\frac{4\left(2^{i}\right)-5 / 3}{15\left(2^{i}\right)-6}$.

Proof. We first construct a rooted graph $R_{i}$ for $i \geq 0$. Then $Q_{i}$ will be built from three disjoint copies of $R_{i}$ by adding a 3-cycle on the roots. With $v$ denoting the root of $R_{i}$, let $R_{i}^{\prime}=R_{i}-v$. We construct $R_{i}$ with $n_{i}$ vertices such that

1. $n_{i}=15\left(2^{i}\right)-6$ and $R_{i}$ is connected,

2. $R_{i}$ decomposes into a 2 -factor on $R_{i}^{\prime}$ and $n_{i} / 3$ disjoint triangles, and

3. $\alpha\left(R_{i}^{\prime}\right)=4\left(2^{i}\right)-2$, with a maximum independent set avoiding the neighbors of $v$.

We show $R_{0}$ in Figure 4 with root $c_{3}$. This graph is connected, has $15\left(2^{0}\right)-6$ vertices, and is the union of a 2-factor on $R_{0}^{\prime}$ and triangles with vertex sets $T_{a}, T_{b}$, and $T_{c}$. An independent set in $R_{0}^{\prime}$ has at most one vertex from each 4-clique, and $\left\{a_{1}, b_{3}\right\}$ is an independent set of size 2 avoiding $T_{c}$, so $\alpha\left(R_{0}^{\prime}\right)=4\left(2^{0}\right)-2=2$.

For $i \geq 1$, start with two disjoint copies of $R_{i-1}$, having roots $c_{3}$ and $d_{3}$. Add triples $T_{x}$ and $T_{y}$ on six new vertices. Augment the union of the 2 -factors in the copies of $R_{i-1}^{\prime}$ 

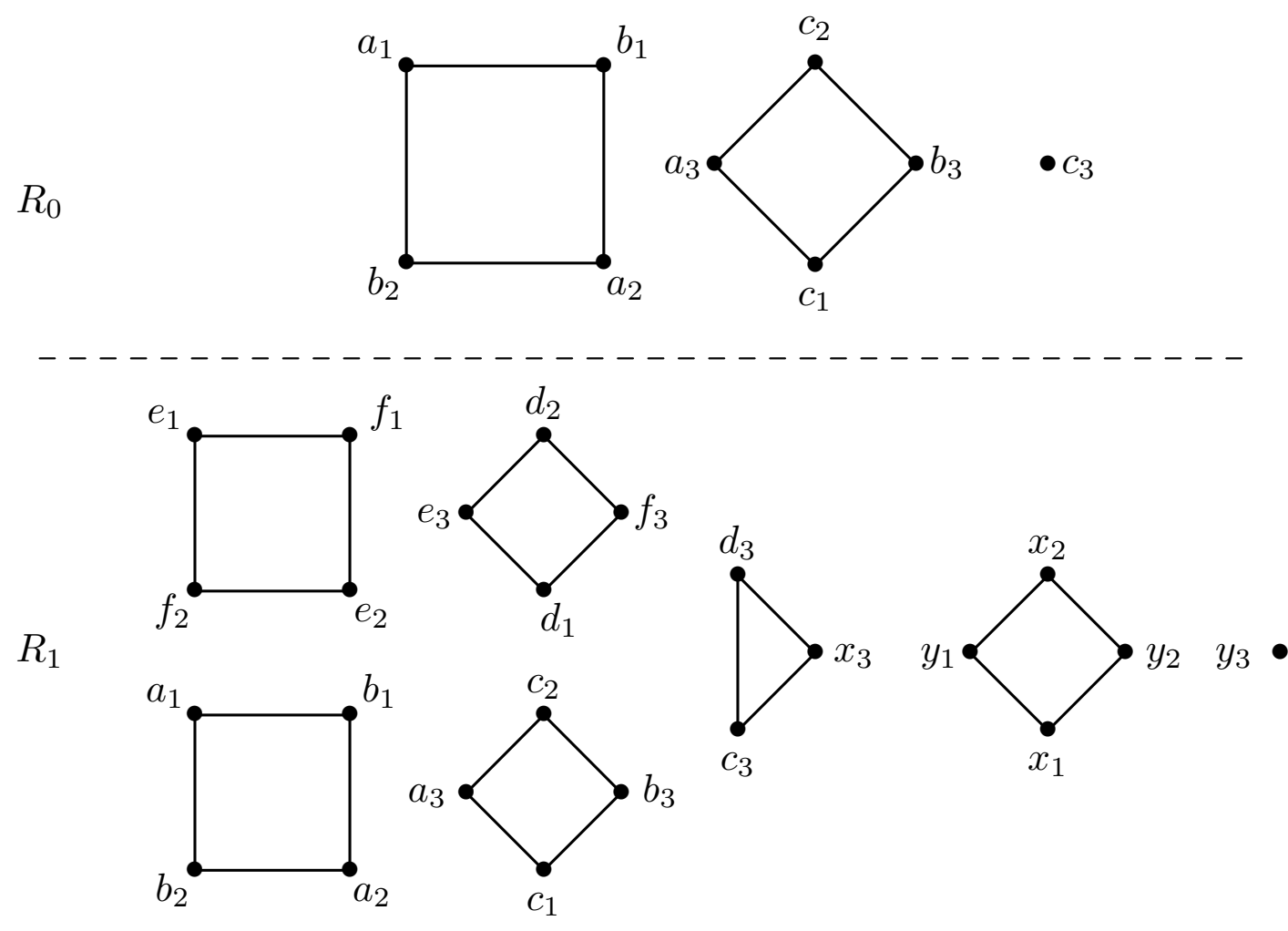

Figure 4: The graphs $R_{0}$ and $R_{1}$

by adding the 3 -cycle $\left[c_{3}, d_{3}, x_{3}\right]$ and the 4 -cycle $\left[x_{1}, y_{1}, x_{2}, y_{2}\right]$. Leave $y_{3}$ as the root in the resulting graph $R_{i}$. Figure 4 shows $R_{1}$.

Doubling and adding six vertices shows inductively that $n_{i}=15\left(2^{i}\right)-6$. By construction, $R_{i}$ is the union of a 2 -factor on $R_{i}^{\prime}$ and $n_{i} / 3$ disjoint triangles. For connectedness, note that inductively each vertex in a copy of $R_{i-1}$ has a path to its root, and using the added 3-cycle, 4-cycle, and triples yields a path from each vertex to the root of $R_{i}$.

It remains to check property (3). Let $I$ be an independent set in $R_{i}^{\prime}$. Maximizing the contributions to $I$ from the two copies of $R_{i-1}^{\prime}$ yields $|I| \leq 2 \alpha\left(R_{i-1}^{\prime}\right)+2=4\left(2^{i}\right)-2$. Furthermore, since $R_{i-1}^{\prime}$ has a maximum independent set avoiding the neighbors of the root of $R_{i-1}$, we can use $c_{3}$ and $x_{1}$ as the two added vertices from $R_{i}^{\prime}$, thereby forming a maximum independent set in $R_{i}^{\prime}$ that avoids $T_{y}$.

In forming $Q_{i}$ by adding a 3-cycle on the roots of three disjoint copies of $R_{i}$, we obtain a connected 2-factor-plus-triangles graph. We can obtain maximum contribution from the three copies of $R_{i}^{\prime}$ obtained by deleting the roots without using any neighbor of the roots. Hence $\alpha\left(Q_{i}\right)=3 \alpha\left(R_{i}^{\prime}\right)+1=12\left(2^{i}\right)-5$. With $Q_{i}$ having $3 n_{i}$ vertices, we obtain the independence ratio claimed. 
In light of Erdös' question concerning the 3-colorability of graphs in $\mathcal{G}$ when 4-cycles are excluded from $G_{1}$, it is natural to ask whether this additional condition guarantees independence ratio $1 / 3$. The answer is no. For every odd $g$, we construct infinitely many graphs in $\mathcal{G}^{\prime}$ with independence ratio less than $1 / 3$ formed using a 2 -factor that has girth $g$. When $g \equiv 1 \bmod 6$, the smallest graph in our family has $g^{2}+2 g$ vertices; this suggests the following conjecture, which by our construction would be asymptotically sharp.

Conjecture 3.3. Every n-vertex graph in $\mathcal{G}^{\prime}$ with girth at least $\sqrt{n}$ has an independent set of size at least $n / 3$.

Our construction was motivated by an arrangement of triples on a 7-cycle, where two of the triples have one element off the cycle. This arrangement, shown in Figure 5, is due to Sachs (see [6]). We use it to build examples with girth 7 . For larger $g$ congruent to 1 modulo 6, we construct an arrangement on a $g$-cycle. A special list allows us to enlarge the arrangement by multiples of 6 .

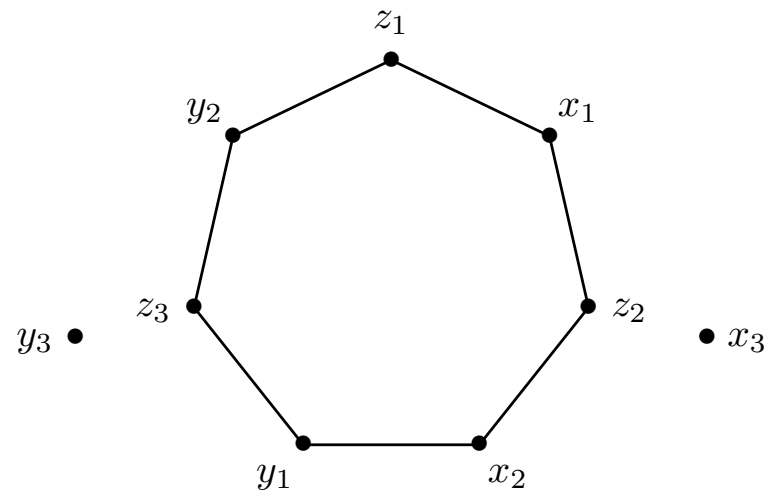

Figure 5: The graph $H_{7}^{\prime}$

Definition 3.4. An $a, b$-brick is a list of six characters plus two holes called notches: $\left(a_{1}, \square, b_{1}, a_{2}, b_{2}, a_{3}, \square, b_{3}\right)$. An $a, b$-brick can link to a $c, d$-brick by starting the $c, d$-brick at the second notch in the $a, b$-brick. The last element of the $a, b$-brick fits into the first notch in the $c, d$-brick. The link leaves notches in the second and next-to-last positions.

A starter brick is a list of seven characters plus two notches that has the form $\left(y_{1}, \square, y_{2}, z_{1}, x_{1}, z_{2}, x_{2}, \square, z_{3}\right)$. For $g=6 j+1$, let $H_{g}^{\prime}$ consist of two special vertices $x_{3}$ and $y_{3}$ plus the cycle of length $g$ whose vertices in order are named by a cyclic arrangement having a starter brick and $a^{(i)}, b^{(i)}$-bricks for $1 \leq i \leq j-1$, linked together in order. The $a^{(1)}, b^{(1)}$-brick links to the second notch of the starter brick, and the $a^{(j-1)}, b^{(j-1)}$-brick links at its end to the first notch of the starter brick. In the degenerate case $j=1$, the starter brick links to itself, producing the graph $H_{7}^{\prime}$ shown in Figure 5. For each symbol $q$, the vertices of $\left\{q_{1}, q_{2}, q_{3}\right\}$ in $H_{g}^{\prime}$ form a triangle. Note that $H_{g}^{\prime}$ has $g+2$ vertices.

The remaining theorems in this section rest on the following simple lemma. 
Lemma 3.5. Let $I$ be an independent set intersecting triples $T_{a}$ and $T_{b}$ in a graph $G$ in $\mathcal{G}$. If $T_{a}$ and $T_{b}$ form an $a, b$-brick in $G_{1}$, and $I$ contains the vertex in a notch of the $a, b$-brick, then I also contains the vertex farthest from it in the a,b-brick.

Proof. An $a, b$-brick has the form $\left(a_{1}, \square, b_{1}, a_{2}, b_{2}, a_{3}, \square, b_{3}\right)$. If $I$ contains the vertex in the first notch, then $I$ omits $a_{1}$ and $b_{1}$. Since $I$ must intersect $T_{a}$, we have $b_{2} \notin I$. Hence $I$ must contain $b_{3}$ to intersect $T_{b}$.

Theorem 3.6. For each odd $g$, there are in $\mathcal{G}^{\prime}$ infinitely many graphs with girth $g$ whose independence ratio is less than $1 / 3$.

Proof. First suppose that $g=6 j+1$. For $k \geq 1$, we construct such a graph $H_{g, h, k}$ with $(g+2) h k$ vertices. Start with $h k$ copies of the graph $H_{g}^{\prime}$ of Definition 3.4, where $h$ is odd and at least 3 . The vertices having the three subscripted copies of a given label form a triple, with $x_{3}$ and $y_{3}$ lying outside the cycle as in Figure 5. Each copy of $H_{g}^{\prime}$ requires an additional superscript in the labels to distinguish its vertices from those of other copies.

Number the copies 0 through $h k-1$. For $0 \leq i \leq k-1$, add a cycle on the vertices representing $x_{3}$ in copies $h i+1$ through $h i+h(\bmod h k)$ of $H^{\prime}$, and add a cycle on the vertices representing $y_{3}$ in copies $h i$ through $h i+h-1$ of $H^{\prime}$. This completes the graph $H_{g, h, k}$; note that it has $(g+2) h k$ vertices and is a 2 -factor-plus-triangles graph.

Since $H_{g}^{\prime}$ has an $x_{3}, y_{3}$-path, the cycles on the copies of $x_{3}$ and $y_{3}$ make it possible to reach each copy of $H^{\prime}$ from any other. Hence $H_{g, h, k}$ is connected.

Each cycle in the 2-factor forming $H_{g, h, k}$ has length $g$ or $h$. A cycle of length $h$ contributes at most $(h-1) / 2$ vertices to an independent set; we apply this to the cycles through the copies of $x_{3}$ and $y_{3}$. There are $2 k$ such cycles, contributing at most $k(h-1)$ vertices. In addition, we claim that the $g$-cycle in each copy of $H_{g}^{\prime}$ contributes at most $2 j$ vertices to an independent set; note that $2 j=(g-1) / 3$. If this claim is true, then

$$
\alpha\left(H_{g, h, k}\right) \leq h k \frac{g-1}{3}+k(h-1)=h k \frac{g+2}{3}-k<h k \frac{g+2}{3}=\frac{1}{3}\left|V\left(H_{g, h, k}\right)\right| .
$$

The inequality would be too weak if the $g$-cycle could contribute $2 j+1$ vertices.

To prove the claim, note that the $g$-cycle contains the vertices of $2 j-1$ full triples (including one in the starter brick) plus $\left\{x_{1}, x_{2}, y_{1}, y_{2}\right\}$. To contribute more than $2 j$ vertices, we must find an independent set having an element from each full triple, plus one of $\left\{x_{1}, x_{2}\right\}$ and one of $\left\{y_{1}, y_{2}\right\}$.

Suppose that such an independent set $I$ exists. Since the last vertex of each brick fits into the first notch of the next brick, $z_{3} \in I$ implies $b_{3}^{(j-1)} \in I$, and $y_{1} \in I$ implies $a_{1}^{(1)} \in I$, by applying Lemma 3.5 iteratively to each ordinary brick. In the first case, $b_{3}^{(j-1)} \in I$ forbids having a vertex from $\left\{y_{1}, y_{2}\right\}$. In the second case, $x_{2}, z_{3} \notin I$, and $I$ cannot have two elements in $\left\{z_{1}, x_{1}, z_{2}\right\}$. Both arguments apply in degenerate form when $k=0$.

In the remaining case, $z_{3}, y_{1} \notin I$. Here one from each of $T_{x}, T_{y}, T_{z}$ must be chosen nonconsecutively from the string $\left(y_{2}, z_{1}, x_{1}, z_{2}, x_{2}\right)$, and this is not possible. This completes the argument for $g \equiv 1 \bmod 6$. 
When $g \not \equiv 1(\bmod 6)$, we set $h$ to be $g$ and let the first value higher than $g$ that is congruent to 1 modulo 6 play the role of $g$ in the construction above. Since $k$ is arbitrary, the family is still infinite.

To form the smallest example constructed in Theorem 3.6 when $g \equiv 1 \bmod 6$, set $h=g$ and $k=1$. The resulting graph $H_{g, g, 1}$ has girth $g$ and has $g^{2}+2 g$ vertices. Letting $n=\left|V\left(H_{g, g, 1}\right)\right|$, we have an $n$-vertex example where $G_{1}$ has girth $\sqrt{n+1}-1$ and the independence ratio ( of $H_{g, g, 1}$ ) is less than $1 / 3$. When $g \not \equiv 1 \bmod 6$ and we must use $H_{g^{\prime}}^{\prime}$ for some $g^{\prime}$ larger than $g$, we use even more vertices. This motivates Conjecture 3.3.

Although girth at least $\sqrt{n}$ in $G_{1}$ may be enough to force an independent set of size $n / 3$ in $G$, it does not force 3-colorability. Surprisingly, no threshold for the girth in terms of $n$ forces this except $n$ itself, where $G$ becomes a cycle-plus-triangles graph. Note that if the girth of an $n$-vertex 2-regular graph $G_{1}$ is not $n$, then it is at most $n / 2$.

Theorem 3.7. If $n=24+12 k$ with $k \geq 0$, then there is an $n$-vertex 2-factor-plus-triangles graph $G$ such that $G_{1}$ consists of two $n / 2$-cycles and $G$ is not 3-colorable.

Proof. We use $a^{(i)}, b^{(i)}$-bricks as in Theorem 3.6, but for this theorem the starter bricks have 12 symbols plus two notches. We use two starter bricks:

$$
\begin{aligned}
& \left(z_{1}, \square, z_{2}, u_{1}, z_{3}, u_{2}, v_{3}, w_{3}, y_{2}, x_{3}, y_{1}, x_{2}, \square, y_{3}\right) \\
& \left(\hat{z}_{2}, \square, \hat{z}_{3}, v_{1}, w_{1}, \hat{z}_{1}, v_{2}, w_{2}, u_{3}, \hat{y}_{2}, x_{1}, \hat{y}_{3}, \square, \hat{y}_{1}\right)
\end{aligned}
$$

Let $G_{1}$ consist of cycles $C$ and $\hat{C}$, where $C$ consists of the first starter brick and $a^{(i)}, b^{(i)_{-}}$ bricks for $1 \leq i \leq k$, and $\hat{C}$ consists of the second starter brick and $\hat{a}^{(i)}, \hat{b}^{(i)}$-bricks for $1 \leq i \leq k$, linked in order as in Theorem 3.6. The triples for $u, v, w, x$ create connections between the two cycles, but all other triples are confined to $C$ or to $\hat{C}$. When $k=0$, each starter brick links into itself to form a 12-cycle. (Examples with $n$ vertices and girth $n / 2-6 r$ arise by using $k-r$ ordinary bricks in $C$ and $k+r$ ordinary bricks in $\hat{C}$; the same argument applies.

Suppose that the resulting graph $G$ has a proper 3-coloring $f$. Each color class is an independent set having one vertex in each triple. Simplifying notation, let $b_{3}$ and $a_{1}$ denote the vertices in the first and second notches of the starter brick in $C$, respectively, while $\hat{b}_{3}$ and $\hat{a}_{1}$ denote those vertices in $\hat{C}$. Without loss of generality, we may assume that $f\left(a_{1}\right)=1$. Repeatedly applying Lemma 3.5 yields $f\left(z_{1}\right)=1$. Now we may assume that $f\left(b_{3}\right)=3$; repeatedly applying Lemma 3.5 yields $f\left(y_{3}\right)=3$.

If the neighbors in $G_{1}$ of a vertex $\alpha$ belong to the same triple, then the third member of that triple must have the same color as $\alpha$. Hence $f\left(x_{3}\right)=f\left(y_{3}\right)=3$, and $f\left(u_{1}\right)=$ $f\left(z_{1}\right)=1$. Also, if a vertex next to $\alpha$ and another member of the triple containing $\alpha$ have distinct colors, then $f(\alpha)$ is the third color. Hence $f\left(x_{2}\right)=2$ and $f\left(z_{2}\right)=2$. Once we color two members of a triple, the third has the third color. Hence $f\left(x_{1}\right)=1$ and $f\left(z_{3}\right)=3$. If two neighbors of $\alpha$ have distinct colors, then $\alpha$ has the third color. Hence $f\left(y_{1}\right)=1$. Now $f\left(y_{2}\right)=2$. 
Since $f\left(z_{3}\right)=3$ and $f\left(u_{1}\right)=1$, we have $f\left(u_{2}\right)=2$, and then $f\left(u_{3}\right)=3$. Now $f\left(x_{1}\right)=1$ and $f\left(u_{3}\right)=3$ imply $f\left(\hat{y}_{2}\right)=2$, and hence $f\left(\hat{y}_{3}\right)=3$ and $f\left(\hat{y}_{1}\right)=1$. This leaves $f\left(\hat{a}_{1}\right)=2$. Iterating Lemma 3.5 now yields $f\left(\hat{z}_{2}\right)=2$ and $f\left(\hat{b}_{3}\right)=1$. Now $f\left(\hat{z}_{3}\right)=3$ and $f\left(\hat{z}_{1}\right)=1$.

We have now determined the colors of all vertices in the starter bricks except those in the triples $T_{v}$ and $T_{w}$. For all other vertices in these bricks, the color matches the subscript. The relevant remaining segments are $\left(u_{2}, v_{3}, w_{3}, y_{2}\right)$ and $\left(\hat{z}_{3}, v_{1}, w_{1}, \hat{z}_{1}, v_{2}, w_{2}, u_{3}\right)$. Color 2 is forbidden from $\left\{v_{3}, w_{3}\right\}$. Hence it appears on one of $\left\{v_{1}, v_{2}\right\}$ and one of $\left\{w_{1}, w_{2}\right\}$. However, the subscripts on its appearances differ. If $f\left(v_{1}\right)=f\left(w_{2}\right)=2$, then $f\left(w_{1}\right)=$ $f\left(v_{2}\right)=3$ (since $f\left(\hat{z}_{1}\right)=1$ ), and then $f\left(v_{3}\right)=f\left(w_{3}\right)$. If $f\left(v_{2}\right)=f\left(w_{1}\right)=2$, then $f\left(w_{2}\right)=f\left(v_{1}\right)=1$ (since $f\left(\hat{z}_{3}\right)=f\left(u_{3}\right)=3$ ), and again $f\left(v_{3}\right)=f\left(w_{3}\right)$. Hence the coloring cannot be completed.

\section{Triangles-Plus-Triangles Graphs}

Although some 2-factor-plus-triangles graphs are not 3-colorable, some (such as cycleplus-triangles graphs) are 3-choosable. Another such class occurs at the other "extreme", when the cycles in the 2-factor are 3-cycles. That is, the union of two graphs on the same vertex set whose components are all triangles is 3 -choosable.

We prove a more general statement in terms of the numbers of vertices in the components of two subgraphs whose union is $G$. Our main tool is the theorem of Galvin [7] about list coloring of the line graphs of bipartite graphs: if $G$ is a bipartite multigraph with maximum degree $k$, then the line graph of $G$ is $k$-choosable.

Proposition 4.1. If $G_{1}$ and $G_{2}$ are graphs whose components have at most s vertices, then $G_{1} \cup G_{2}$ is s-choosable.

Proof. Let $G=G_{1} \cup G_{2}$. By adding isolated vertices to $G_{1}$ and/or $G_{2}$ as needed, we may assume that $V\left(G_{1}\right)=V\left(G_{2}\right)=V(G)$ without changing $G$. For each $v \in V(G)$, let $L(v)$

be a set of $s$ available colors. Form a graph $H$ with a vertex for each component of $G_{1}$ and a vertex for each component of $G_{2}$. For each vertex of $G$, place an edge in $H$ joining the vertices representing the components containing it in $G_{1}$ and $G_{2}$ ( $H$ is the "intersection graph" of the components in $G_{1}$ and $G_{2}$ ). By construction, $H$ is bipartite. The degree of a vertex in $H$ is the number of vertices in the corresponding component of $G_{1}$ or $G_{2}$.

Each edge of $H$ corresponds to a vertex $v$ in $G$. Assign to this edge the list $L(v)$. Since $H$ is bipartite and has maximum degree at most $s$, Galvin's Theorem implies that we can choose a proper edge-coloring of $H$ from the lists. This assigns colors to the vertices of $G$ from their lists so that vertices in the same component of $G_{1}$ or in the same component of $G_{2}$ have distinct colors. Hence it is a proper coloring of $G$.

In particular, every triangles-plus-triangles graph is 3-choosable. 


\section{References}

[1] D.-Z. Du, D. F. Hsu, and F. K. Hwang, The Hamiltonian property of consecutive-d digraphs, in Graph-theoretic models in computer science, II (Las Cruces, NM, 19881990), Mathematical and Computer Modelling, 17 (1993), 61-63.

[2] D.-Z. Du, and H. Q. Ngo, An extension of DHH-Erdős conjecture on cycle-plus-triangle graphs, Taiwanese J. Math., 6 (2002), 261-267.

[3] P. Erdös, On some of my favourite problems in graph theory and block designs, in Graphs, designs and combinatorial geometries (Catania, 1989), Le Matematiche, 45 (1990), 61-73 (1991).

[4] P. Erdős, A. L. Rubin, and H. Taylor, Choosability in graphs Proc. West Coast Conf. on Combinatorics and Computing (Humboldt State Univ., Arcata, Calif., 1979), Congressus Numerantium 26 (1980), 125-157.

[5] H. Fleischner and M. Stiebitz, A solution to a colouring problem of P. Erdős, Special volume to mark the centennial of Julius Petersen's "Die Theorie der regulären Graphs", Part II, Discrete Mathematics, 101 (1992), 39-48.

[6] H. Fleischner and M. Stiebitz, Some remarks on the cycle plus triangles problem, in The mathematics of Paul Erdös, II (Springer), Algorithms and Combinatorics, 14 (1997), 136-142.

[7] F. Galvin, The list chromatic index of a bipartite multigraph, J. Combin. Theory (B), 63 (1995), 153-158.

[8] H. Sachs, Elementary proof of the cycle-plus-triangles theorem, in Combinatorics, Paul Erdős is eighty, Vol. 1, Bolyai Soc. Math. Stud., (János Bolyai Math. Soc., 1993), $347-$ 359 . 